\title{
The Factors Influencing Job Burnout Among Coal Miners in Xinjiang Uygur Autonomous Region,China: a Cross-Sectional Study
}

\section{Huijun Deng}

Xinjiang Medical University

\section{Xinnan Li}

People's Hospital of Xinjiang Uygur Autonomous Region

\section{Xieerwaniguli Abulimiti}

Xinjiang Medical University

\section{Zulipinu Mutailifu}

Xinjiang Medical University

\section{Shuaiyin Zheng}

Xinjiang Medical University

Xin Lin

Xinjiang Medical University

Fuye Li ( $\square$ lifuye2000@163.com )

Xinjiang Medical University https://orcid.org/0000-0003-3621-0539

\section{Research article}

Keywords: coal miners, job burnout, occupational stress, work-family-conflict, effort-reward imbalance

Posted Date: April 1st, 2021

DOl: https://doi.org/10.21203/rs.3.rs-368800/v1

License: (c) (i) This work is licensed under a Creative Commons Attribution 4.0 International License. Read Full License 


\section{Abstract}

Background: Coal miners are prone to burnout symptoms due to their special working environment. There are no studies on the correlation between job burnout and occupational stress, effort-reward imbalance, and work-family-conflict.

Methods: In this cross-sectional study, 1,346 coal miners were selected from 5 coal mining companies using stratified cluster sampling method. The Chinese version of the Job Burnout Scale, Job Content Questionnaire (JCQ), Effort-Reward Inventory (ERI), and Working-Family-Conflict Scale were used to collect data from the coal miners. Correlation analysis and logistic regression methods were used to explore the factors affecting job burnout.

Results: coal miners with different genders, ages, years of service, shift system, marital status, and type of work have different total scores and levels of job burnout $(P<0.05)$. The effort-reward imbalance is associated with age, years of service, shift system and marital status $(P<0.05)$. The occupational stress scores of coal miners of different genders, years of service, education, shift system and monthly income were different. Different shift systems, types of work, and work-family conflict scores were also different. The various dimensions of job burnout have varying degrees of correlation with the JCQ, ERI, and Working-Family-Conflict dimensions.Age, work demands, effort, work-family conflict and work-family conflict are risk factors for job burnout, and work autonomy is the protective factor.

Conclusions:The detection rate of job burnout in coal miners in Xinjiang Uygur Autonomous Region is relatively high. Age, work demands, effort, and work-family conflict increase the incidence of job burnout, while work autonomy reduces job burnout.

\section{Background}

As a social psychological factor, job burnout was first diagnosed in 1974 by a New York-based clinical psychologist $\mathrm{H}$. J. Freudenberger suggesting [1] that it is a syndrome of emotional and physical exhaustion triggered by work-related stress [2].Maslach C scholars divide it into three dimensions: emotional exhaustion (mental fatigue, physical and mental fatigue), depersonalization (negativity, indifference), and personal accomplishment (lack of work feeling)[3].Due to rapid economic development, the high rates of job burnout are common in various industries. The incidence of job burnout among nursing staff and the general population in the West is about $20-30 \%[4,5]$, Middle East and Africa About $40-80 \%$ of medical staff[6, 7]. Job burnout is closely associated with job satisfaction, anxiety and depression [8]. In recent years, work pressure and tension have increased dramatically in China, and job burnout has also been relatively serious [9]. At present, the coal mines in China account for about 13.9\% of the total coal mines in the world. There are nearly 4 million people engaged in underground coal mine operations [10]. Coal miners are a special group of professionals who are exposed to poor mining conditions ( Dim underground, narrow working area, danger of landslides, high temperature and humidity, noise, vibration, etc.), strict production requirements and high incidence of occupational injuries 
(pneumoconiosis, musculoskeletal diseases, etc.), this results in employees with serious psychological problems such as depression [11], and severe job burnout [12], which makes the miners prone to accidents [13]. This harms the benefits of organization and society, and also endangers the production and safety of miners [14]. Work-family-conflict is a concept that was first proposed by Greenhouse and others in 1985 in which professional workers cannot balance the roles of work and family. It is reported that work-family-conflict is an important factor affecting job burnout, especially working time conflict [15, 16]. The imbalance between work efforts and work rewards also causes an increase in job burnout [17, 18].Occupational stress is an inappropriate psychological pressure caused by employees in the work environment because their job requirements and ability requirements exceed their personal capacity, and it is more closely related to job burnout $[14,9]$. Xinjiang is one of the main coal mining regions in China, yet there are few studies on the relationship between job burnout and effort-reward imbalance, workfamily-conflict, and occupational stress among coal mine workers. Therefore, this study aims to investigate the link among these factors and their association with job burnout. The purpose is to fully understand the job burnout of coal miners and provide relevant evidence for occupational health management and intervention of coal miners.

\section{Objectives}

The specific objectives of this study are as follows: (i) Investigate the status of job burnout among coal miners in the Xinjiang Uygur Autonomous Region. (ii) Verify whether occupational stress, effort-reward imbalance and work-family-conflict are associated with job burnout. (iii) Explore the factors influencing job burnout among coal miners.

\section{Methods}

\section{Study population}

Ethical approval for this study was granted by the Ethical Committee of the First Affiliated Hospital of Xinjiang Medical University(No.20160218-109). Random sampling was used to select 5 coal mining enterprises (the annual output of coal is divided into three layers: large, medium and small.One large, two medium, and two small companies are selected: Heishan 839, Baoying 178, Xiaogangou 155, Yan mine 97, Yutian 77) in Xinjiang.The inclusion criteria include: (1) informed consent and voluntary participation in this survey; (2) formal employment in a coal mine; (3) 18 years old $\leq$ age $<60$ years old; length of service $\geq 1$ year; (4) no physical examination, mental illness and genetic history; and (5) no blood relationship between the subjects. On the other hand, the exclusion criterion was when nearly $20 \%$ of the data were incomplete. Due to the lack of data on the prevalence of job burnout in China, the prevalence rate of this study refers to the prevalence rate of $27.5 \%$ of job burnout among 8028 occupational groups in various industries surveyed in the UK in 2006. According to the current research sample size

calculation formula ${ }^{n=\frac{Z_{x}{ }^{2} \times p q}{d^{2}}}$ 
( $Z a=1.96$, significance level $a=0.05, p=27.5 \%, q=1-p$, allowable error $d=0.1 p)$, the sample size $n=1013$ is obtained. Considering the loss of follow-up rate of $20 \%$, the required sample size is at least 1,266 .A total of 1,500 questionnaires were distributed in this survey, and 154 unqualified questionnaires were eliminated. In the end, 1,346 valid questionnaires were recovered, with a recovery rate of $89.73 \%$.

\section{Research methods}

Self-designed general survey囚including the age, gender, ethnicity, education, length of service, type of work, marital status of coal miners.

\section{Job burnout}

The measurement of job burnout was based on the newly developed Dai Junming's job burnout questionnaire by Fuye Li. Notably, the scale harbors a satisfactory reliability and validity with its retest reliability being 0.843 and Cronbach a Coefficient was $0.780[19,20]$. The scale included 3 dimensions (emotional exhaustion, depersonalization, and personal accomplishment) and 15 questions (5 questions for each dimension). The questionnaire was assigned to Likert level 7. The range from 1 to 7 represented "never" to "every day", and the personal accomplishment dimension $(3,6,9,12,15$ questions) were scored in reverse order. Based on 25 scores for emotional exhaustion, 11 scores for depersonalization, and 16 scores for personal achievement, job burnout levels were divided into no burnout (all below 3 thresholds), mild burnout (only above 1 threshold), moderate burnout (only above 2 thresholds), and severe burnout (all above 3 thresholds) .

\section{Occupational stress}

The Job Content Questionnaire (JCQ) is a concise occupational stress questionnaire based on the model of "work demands-work autonomy-social support". Other domestic studies have confirmed that the questionnaire has good reliability and validity[21,22]. The questionnaire consists of three modules, 5 items for work demands; 9 items for work autonomy (decision autonomy, working ability); and 8 items for social support (superior support, colleague support), totaling 22 items. The questionnaire uses Likert level 5 scoring.

\section{Effort-Reward Imbalance}

The Effort-Reward Imperfection Scale (ERI) includes three modules, which are External contribution (6 items), work reward (11 items), and internal investment (6 items). The questionnaire uses Likert level 5 score, from " 1 " to " 5 " representing the degree of conformity from "not at all" to "always will"; ERI=external contribution $(E) /$ work reward $(R) \times 11 / 6$. ERI $<1$ indicates a state of low efforts-high reward, while $E R I>1$ indicates a state of high efforts-low reward. The larger the ERI ratio, the higher the imbalance of efforts and reward.

\section{Work-family-conflict}


This study adopted the work-family-conflict scale revised by Carlson in 2000 [23], which includes two parts: work-family conflict and family-work conflict. Each part is scored separately based on time, pressure and behavior. Each module includes 9 items, totaling 18 items. The questionnaire uses Likert level 5scoring, from " 1 " to " 5 " representing the degree of conformity from "strongly disagree" to "strongly agree". The higher the score, the stronger the conflict.

\section{Statistical Analysis}

The investigators who had undergone similar training and assessment issued a survey form with instructions and informed consent to the survey subjects. The information collected from the questionnaire was verified by the duo and entered into the Epidata 3.1 database. After verifying the consistency of data the 21.0 version of IBM SPSS Statistics software was used to analyze the data. Quantitative data are statistically described by mean \pm standard deviation. Two independent sample ttests were used for comparison of two groups of means, and single factor was used for comparison of means for multiple groups. In the analysis of variance, if there is a difference in the population, the SNK-q test was used for pairwise comparison. The categorical variables were evaluated using the $\chi^{2}$ test. Single factor and Logistic regression analysis were used to determine the factors influencing job burnout. Bilateral test level $\mathrm{a}=0.05$. $P<0.05$ was considered to be statistically significant.

\section{Results}

\section{Comparison of different demographic characteristics of coal miners}

A total of 1,346 coal mine workers which includes 1,293 men (96.1\%) and 53 women (3.9\%) completed the questionnaire for this study. The average score for job burnout among the coal mine workers was $53.74 \pm 10.64$ points, while the average score for occupational stress was $50.78 \pm 11.93$ points. The average score for work-family-conflict was $49.45 \pm 10.91$ points, while 641 people recorded high effortlow reward.

The total scores for job burnout among the coal miners differed among the different genders, ages, length of service, education, shift system, marital status, and types of work. Male workers had higher scores than female workers, while workers between the age of 30 to 45 recorded the highest scores among all the age groups. Similarly, workers who had worked for $\geq 11$ years recorded higher scores than those who had worked for 1-10 years. The higher the level of education, the lower the score for job burnout, while workers who worked in shifts recorded higher scores than workers who worked day shift. Lastly, the score for workers who were married/divorced was higher than that of workers who were single $(P<0.05)$.

The results of occupational stress analysis also showed that the scores differed among different genders, length of service, education, shift system and monthly income. Male workers also had higher 
scores than female workers, while workers who had worked for $\geq 11$ years had higher scores than those who had worked for 1-10 years. The higher the level of education the higher the score for occupational stress, while the workers who worked day shift recorded higher scores than those who worked in shifts. Miners with a monthly income $>7000$ recorded higher scores than those earning $<7000(P<0.05)$.

An analysis of the imbalance between effort and reward showed that 159 people $(11.81 \%)$ have ERI $\leq 1$ while 1187 people (88.19\%) have ERI $>1$. The occurrence of the imbalance between effort and reward was associated with age, length of service and marital status $(P<0.05)$, but had no association with gender, education, shift system, type of work and other factors $(P>0.05)$.

The results of work-family-conflict analysis showed that the scores differed among different shift systems, types of work. Shift miners score higher than day shifts; coal miners and excavate miners scores higher compared with other types of work $(P<0.05)$;And had no association with gender, length of service, education and monthly income $(P>0.05)$ (Table 1$)$. 
Table 1

Comparison of job burnout, occupational stress, unbalanced efforts and reward, and work-family-conflict among coal miners with different demographic characteristics $(n=1346)$

\begin{tabular}{|c|c|c|c|c|c|}
\hline Variable & $N$ & $\begin{array}{l}\text { Job } \\
\text { burnout }\end{array}$ & $\begin{array}{l}\text { Occupational } \\
\text { stress }\end{array}$ & $E R I>1$ & $\begin{array}{l}\text { work- } \\
\text { family- } \\
\text { conflict }\end{array}$ \\
\hline \multicolumn{6}{|l|}{ Gender } \\
\hline male & 1293 & $\begin{array}{l}53.87 \pm \\
10.55\end{array}$ & $71.22 \pm 10.38$ & $622(97.0 \%)$ & $\begin{array}{l}49.57 \pm \\
10.92\end{array}$ \\
\hline female & 53 & $\begin{array}{l}50.60 \pm \\
12.82\end{array}$ & $68.28 \pm 10.06$ & $19(3.0 \%)$ & $\begin{array}{l}46.57 \pm \\
10.70\end{array}$ \\
\hline$t / \chi^{2}$ & & 2.187 & 2.023 & 3.066 & 1.898 \\
\hline$P$ & & 0.029 & 0.043 & 0.080 & 0.580 \\
\hline \multicolumn{6}{|l|}{ Age(years) } \\
\hline $18 \sim 30$ & 359 & $\begin{array}{l}49.65 \pm \\
8.89\end{array}$ & $71.20 \pm 10.49$ & $147(22.9 \%)$ & $\begin{array}{l}50.31 \pm \\
10.59\end{array}$ \\
\hline $30 \sim 45$ & 548 & $\begin{array}{l}55.88 \pm \\
10.08^{\mathrm{a}}\end{array}$ & $70.75 \pm 10.35$ & $246(38.4 \%)$ & $\begin{array}{l}49.33 \pm \\
10.83\end{array}$ \\
\hline $45 \sim 60$ & 439 & $\begin{array}{l}54.41 \pm \\
11.74^{\text {ab }}\end{array}$ & $71.46 \pm 10.32$ & $248(38.7 \%)$ & $\begin{array}{l}48.60 \pm \\
11.26\end{array}$ \\
\hline$F / \chi^{2}$ & & 40.504 & 0.586 & 21.899 & 2.430 \\
\hline$P$ & & $<0.001$ & 0.557 & $<0.001$ & 0.088 \\
\hline \multicolumn{6}{|l|}{ Length of service(years) } \\
\hline $1 \sim 10$ & 986 & $\begin{array}{l}53.00 \pm \\
10.33\end{array}$ & $70.38 \pm 10.48$ & $434(67.7 \%)$ & $\begin{array}{l}49.34 \pm \\
10.98\end{array}$ \\
\hline$\geq 11$ & 360 & $\begin{array}{l}55.76 \pm \\
11.29\end{array}$ & $73.11 \pm 9.83$ & $207(32.2 \%)$ & $\begin{array}{l}49.41 \pm \\
10.77\end{array}$ \\
\hline$t / x^{2}$ & & -4.230 & -4.299 & 19.222 & -0.105 \\
\hline$P$ & & $<0.001$ & $<0.001$ & $<0.001$ & 0.916 \\
\hline \multicolumn{6}{|l|}{ Education } \\
\hline junior high school and below & 823 & $\begin{array}{l}54.42 \pm \\
10.54\end{array}$ & $69.83 \pm 10.07$ & $392(61.2 \%)$ & $\begin{array}{l}49.4 \pm \\
10.70\end{array}$ \\
\hline \multicolumn{6}{|c|}{ 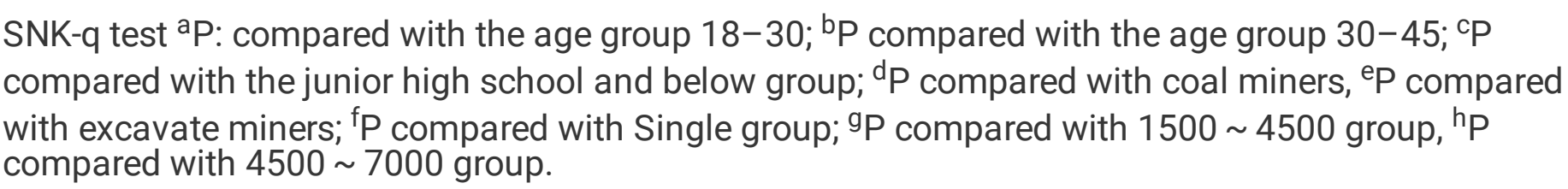 } \\
\hline
\end{tabular}




\begin{tabular}{|c|c|c|c|c|c|}
\hline Variable & $N$ & $\begin{array}{l}\text { Job } \\
\text { burnout }\end{array}$ & $\begin{array}{l}\text { Occupational } \\
\text { stress }\end{array}$ & $E R I>1$ & $\begin{array}{l}\text { work- } \\
\text { family- } \\
\text { conflict }\end{array}$ \\
\hline high school & 361 & $\begin{array}{l}53.29 \pm \\
10.58^{\mathrm{C}}\end{array}$ & $71.99 \pm 10.33^{c}$ & $173(27.0 \%)$ & $\begin{array}{l}49.45 \pm \\
11.35\end{array}$ \\
\hline $\begin{array}{l}\text { bachelor and college degree } \\
\text { and above }\end{array}$ & 162 & $\begin{array}{l}51.26 \pm \\
11.12^{\mathrm{c}}\end{array}$ & $75.57 \pm 10.61^{c}$ & 76(11.9\%) & $\begin{array}{l}48.87 \pm \\
11.12\end{array}$ \\
\hline$F / \chi^{2}$ & & 6.460 & 23.185 & 0.046 & 0.183 \\
\hline$P$ & & 0.002 & $<0.001$ & 0.977 & 0.833 \\
\hline \multicolumn{6}{|l|}{ Type of work } \\
\hline anchor miners & 43 & $\begin{array}{l}53.93 \pm \\
9.27\end{array}$ & $71.23 \pm 9.73$ & $22(3.4 \%)$ & $\begin{array}{l}49.93 \pm \\
10.63\end{array}$ \\
\hline coal miners & 514 & $\begin{array}{l}53.95 \pm \\
10.48\end{array}$ & $70.33 \pm 10.50$ & $258(40.2 \%)$ & $\begin{array}{l}48.64 \pm \\
10.37\end{array}$ \\
\hline excavate miners & 394 & $\begin{array}{l}55.33 \pm \\
10.20^{d}\end{array}$ & $71.85 \pm 9.70$ & $188(29.3 \%)$ & $\begin{array}{l}50.32 \pm \\
10.79\end{array}$ \\
\hline moving frame miner & 69 & $\begin{array}{l}53.43 \pm \\
11.60\end{array}$ & $71.49 \pm 10.18$ & $35(50.7 \%)$ & $\begin{array}{l}46.65 \pm \\
11.43^{d}\end{array}$ \\
\hline other & 326 & $\begin{array}{l}51.52 \pm \\
11.13^{\mathrm{de}}\end{array}$ & $71.33 \pm 11.06$ & $138(21.5 \%)$ & $\begin{array}{l}47.64 \pm \\
11.32^{\mathrm{de}}\end{array}$ \\
\hline$F / \chi^{2}$ & & 5.867 & 1.287 & 5.506 & 4.041 \\
\hline$P$ & & $<0.001$ & 0.273 & 0.239 & 0.003 \\
\hline \multicolumn{6}{|l|}{ Shift system } \\
\hline day shift & 174 & $\begin{array}{l}50.57 \pm \\
12.35\end{array}$ & $74.36 \pm 9.93$ & $80(46.0 \%)$ & $\begin{array}{l}46.78 \pm \\
12.57\end{array}$ \\
\hline shift work & 1172 & $\begin{array}{l}54.21 \pm \\
10.31\end{array}$ & $70.63 \pm 10.36$ & $561(47.9 \%)$ & $\begin{array}{l}49.74 \pm \\
10.61\end{array}$ \\
\hline$t / x^{2}$ & & -4.221 & 4.459 & 0.217 & -3.349 \\
\hline$P$ & & $<0.001$ & $<0.001$ & 0.641 & 0.001 \\
\hline
\end{tabular}

SNK-q test ${ }^{\text {aP: }}$ compared with the age group $18-30$; ${ }^{b} \mathrm{P}$ compared with the age group $30-45$; $^{\mathrm{C}} \mathrm{P}$ compared with the junior high school and below group; ${ }^{\mathrm{d} P}$ compared with coal miners, ${ }^{\mathrm{e}} \mathrm{P}$ compared with excavate miners; ${ }^{\mathrm{P}}$ compared with Single group; ${ }^{\mathrm{P}} \mathrm{P}$ compared with $1500 \sim 4500$ group, ${ }^{\mathrm{h}} \mathrm{P}$ compared with $4500 \sim 7000$ group. 


\begin{tabular}{|c|c|c|c|c|c|}
\hline Variable & $N$ & $\begin{array}{l}\text { Job } \\
\text { burnout }\end{array}$ & $\begin{array}{l}\text { Occupational } \\
\text { stress }\end{array}$ & $E R I>1$ & $\begin{array}{l}\text { work- } \\
\text { family- } \\
\text { conflict }\end{array}$ \\
\hline single & 167 & $\begin{array}{l}49.14 \pm \\
11.25\end{array}$ & $70.89 \pm 11.16$ & $66(10.3 \%)$ & $\begin{array}{l}48.32 \pm \\
10.79\end{array}$ \\
\hline married & 1151 & $\begin{array}{l}54.34 \pm \\
10.46^{f}\end{array}$ & $71.08 \pm 10.27$ & $564(88.0 \%)$ & $\begin{array}{l}49.53 \pm \\
10.97\end{array}$ \\
\hline divorced/separated & 28 & $\begin{array}{l}56.32 \pm \\
8.36^{f}\end{array}$ & $73.32 \pm 9.94$ & $11(1.7 \%)$ & $\begin{array}{l}48.46 \pm \\
9.59\end{array}$ \\
\hline$F / \chi^{2}$ & & 18.701 & 0.678 & 6.051 & 0.977 \\
\hline$P$ & & $<0.001$ & 0.508 & 0.049 & 0.377 \\
\hline \multicolumn{6}{|c|}{ Monthly income(yuan) } \\
\hline $1500 \sim 4500$ & 108 & $\begin{array}{l}53.89 \pm \\
10.69\end{array}$ & $69.45 \pm 10.36$ & $55(8.6 \%)$ & $\begin{array}{l}48.63 \pm \\
11.06\end{array}$ \\
\hline $4500 \sim 7000$ & 805 & $\begin{array}{l}53.718 \pm \\
10.56\end{array}$ & $70.42 \pm 10.15$ & $383(59.8 \%)$ & $\begin{array}{l}49.55 \pm \\
10.99\end{array}$ \\
\hline$>7000$ & 433 & $\begin{array}{l}53.74 \pm \\
10.87\end{array}$ & $\begin{array}{l}72.80 \pm \\
10.59 \mathrm{gh}\end{array}$ & $203(31.7 \%)$ & $\begin{array}{l}49.17 \pm \\
10.75\end{array}$ \\
\hline$F / \chi^{2}$ & & 0.012 & 8.990 & 0.568 & 0.430 \\
\hline$P$ & & 0.988 & $<0.001$ & 0.753 & 0.651 \\
\hline \multicolumn{6}{|c|}{$\begin{array}{l}\text { SNK-q test }{ }^{\text {ap}} \text { : compared with the age group } 18-30 \text {; }{ }^{b} \mathrm{P} \text { compared with the age group } 30-45 ;{ }^{c} \mathrm{P} \\
\text { compared with the junior high school and below group; }{ }^{\mathrm{d}} \mathrm{P} \text { compared with coal miners, }{ }^{\mathrm{e}} \mathrm{P} \text { compared } \\
\text { with excavate miners; }{ }^{\mathrm{f}} \mathrm{P} \text { compared with Single group; }{ }^{\mathrm{g}} \mathrm{P} \text { compared with } 1500 \sim 4500 \text { group, }{ }^{\mathrm{h}} \mathrm{P} \\
\text { compared with } 4500 \sim 7000 \text { group. }\end{array}$} \\
\hline
\end{tabular}

\section{Comparison of job burnout levels of coal miners with different demographic characteristics}

Job burnout was categorized into no burnout, mild burnout, moderate burnout and severe burnout. There were 92 people (6.8\%) with no burnout, 391 people (29.0\%) with mild burnout, 645 people $(47.9 \%)$ with moderate burnout, 218 people (16.2\%) with severe burnout. Results of chi-square analysis showed that the level of job burnout differed among different genders, ages, length of service, shift system, marital status and different types of work $(P<0.05)$. This indicates that the occurrence of job burnout may be associated with the above factors (Table 2 ). 
Table 2

The General situation of burnout grade $(n=1346)$

\begin{tabular}{|c|c|c|c|c|c|c|}
\hline \multirow[t]{2}{*}{ Variable } & \multicolumn{4}{|c|}{ Burnout level } & \multirow[t]{2}{*}{$x^{2}$} & \multirow[t]{2}{*}{$\mathbf{P}$} \\
\hline & $\begin{array}{l}\text { No } \\
\text { burnout }\end{array}$ & $\begin{array}{l}\text { Mild } \\
\text { burnout }\end{array}$ & $\begin{array}{l}\text { Moderate } \\
\text { burnout }\end{array}$ & $\begin{array}{l}\text { Severe } \\
\text { bumout }\end{array}$ & & \\
\hline \multicolumn{7}{|l|}{ Gender } \\
\hline male & $83(6.4 \%)$ & $377(29.2 \%)$ & $622(48.1 \%)$ & $211(16.3 \%)$ & \multirow[t]{2}{*}{8.981} & \multirow[t]{2}{*}{0.030} \\
\hline female & $9(17.0 \%)$ & $14(26.4 \%)$ & $23(43.4 \%)$ & $7(13.2 \%)$ & & \\
\hline \multicolumn{7}{|l|}{ Age(years) } \\
\hline $18 \sim 30$ & $38(10.6 \%)$ & $125(34.8 \%)$ & $186(51.8 \%)$ & $10(2.8 \%)$ & \multirow[t]{3}{*}{75.587} & \multirow{3}{*}{ <. 001} \\
\hline $30 \sim 45$ & $25(4.6 \%)$ & $141(25.7 \%)$ & $296(49.1 \%)$ & $113(20.6 \%)$ & & \\
\hline $45 \sim 60$ & $29(6.6 \%)$ & $125(28.5 \%)$ & 190(43.3\%) & $95(21.6 \%)$ & & \\
\hline \multicolumn{7}{|l|}{$\begin{array}{l}\text { Length of } \\
\text { service(years) }\end{array}$} \\
\hline $1 \sim 10$ & $72(7.3 \%)$ & $304(30.8 \%)$ & $434(67.7 \%)$ & $128(13.0 \%)$ & \multirow[t]{2}{*}{29.444} & \multirow{2}{*}{$<.001$} \\
\hline$\geq 11$ & $20(5.6 \%)$ & $87(24.2 \%)$ & $163(45.3 \%)$ & $90(25.0 \%)$ & & \\
\hline \multicolumn{7}{|l|}{ Education } \\
\hline $\begin{array}{l}\text { junior high school and } \\
\text { below }\end{array}$ & $47(5.7 \%)$ & $299(27.8 \%)$ & $406(49.3 \%)$ & $141(17.1 \%)$ & \multirow[t]{3}{*}{10.015} & \multirow[t]{3}{*}{0.124} \\
\hline high school & $27(7.5 \%)$ & $112(31.0 \%)$ & $165(45.7 \%)$ & $57(15.8 \%)$ & & \\
\hline $\begin{array}{l}\text { bachelor and college } \\
\text { degree and above }\end{array}$ & $18(11.1 \%)$ & $50(30.9 \%)$ & 74(45.7\%) & $20(12.3 \%)$ & & \\
\hline \multicolumn{7}{|l|}{ Type of work } \\
\hline anchor miners & $5(7.2 \%)$ & $27(39.1 \%)$ & $30(43.5 \%)$ & $7(10.1 \%)$ & \multirow[t]{5}{*}{38.097} & \multirow{5}{*}{ <. 001} \\
\hline coal miners & $38(7.4 \%)$ & $129(25.1 \%)$ & $263(51.2 \%)$ & $84(16.3 \%)$ & & \\
\hline excavate miners & $14(3.6 \%)$ & $114(28.9 \%)$ & $181(45.9 \%)$ & $85(21.6 \%)$ & & \\
\hline moving frame miner & $1(2.3 \%)$ & $18(41.9 \%)$ & $19(44.2 \%)$ & $5(11.6 \%)$ & & \\
\hline other & $34(10.4 \%)$ & $103(31.6 \%)$ & $152(46.6 \%)$ & $37(11.3 \%)$ & & \\
\hline \multicolumn{7}{|l|}{ Shift system } \\
\hline day shift & $26(14.9 \%)$ & $52(29.9 \%)$ & $73(42.0 \%)$ & $23(13.2 \%)$ & \multirow[t]{2}{*}{21.844} & \multirow[t]{2}{*}{0.001} \\
\hline shift work & $66(5.6 \%)$ & $339(28.9 \%)$ & $572(48.8 \%)$ & $195(16.6 \%)$ & & \\
\hline
\end{tabular}




\begin{tabular}{|c|c|c|c|c|c|c|}
\hline \multirow[t]{2}{*}{ Variable } & \multicolumn{4}{|c|}{ Burnout level } & \multirow[t]{2}{*}{$x^{2}$} & \multirow[t]{2}{*}{$\mathbf{P}$} \\
\hline & $\begin{array}{l}\text { No } \\
\text { burnout }\end{array}$ & $\begin{array}{l}\text { Mild } \\
\text { burnout }\end{array}$ & $\begin{array}{l}\text { Moderate } \\
\text { burnout }\end{array}$ & $\begin{array}{l}\text { Severe } \\
\text { burnout }\end{array}$ & & \\
\hline single & $22(13.2 \%)$ & $60(35.9 \%)$ & $72(43.1 \%)$ & $13(7.8 \%)$ & \multirow[t]{3}{*}{28.189} & \multirow{3}{*}{$\begin{array}{l}<.001 \\
0.001\end{array}$} \\
\hline married & $69(6.0 \%)$ & $321(27.9 \%)$ & $557(48.4 \%)$ & $204(17.7 \%)$ & & \\
\hline divorced/separated & $1(3.6 \%)$ & $10(35.7 \%)$ & $16(57.1 \%)$ & $1(3.6 \%)$ & & \\
\hline \multicolumn{7}{|c|}{ Monthly income( yuan) } \\
\hline $1500 \sim 4500$ & $8(7.4 \%)$ & $26(24.1 \%)$ & $54(50.0 \%)$ & $20(18.5 \%)$ & \multirow[t]{3}{*}{4.438} & \multirow[t]{3}{*}{0.618} \\
\hline $4500 \sim 7000$ & $53(6.6 \%)$ & $250(31.1 \%)$ & $378(47.0 \%)$ & $124(15.4 \%)$ & & \\
\hline$>7000$ & $31(7.2 \%)$ & $115(26.6 \%)$ & $213(49.3 \%)$ & $74(17.1 \%)$ & & \\
\hline
\end{tabular}

After controlling demographic factors such as length of service, type of work, shift system, and marital status, a partial correlation analysis of job burnout, occupational stress,effort-reward imbalance and work-family-conflict was performed. The results showed that emotional exhaustion was positively correlated with work demands,working ability,efforts, and internal investment $(r=0.273,0.104,0.284$, $0.256 ; P<0.05)$, and negatively correlated with decision autonomy $(r=-0.104 ; P<0.05)$, and no correlation has been found with return $(P>0.05)$;Depersonalization was positively correlated with work demands, efforts, and internal investment $(r=0.162,0.179,0.138)$, and negatively correlated with job skills and returns $(\mathrm{r}=-0.066,-0.128 ; P<0.05)$, no correlation has been found with decision autonomy $(P>$ 0.05);Personal accomplishment is negatively correlated with all dimensions of occupational stress $(r=-0.128,-0.116,-0.166,-0.097,-0.147 ; P<0.05)$, and positively correlated with efforts and internal investment $(r=0.107,0.083 ; P<0.05)$, no correlation has been found with the return $(P>0.05)$.

Emotional exhaustion was positively correlated with work-family conflict $(r=0.179,0.316,0.308)$ and family-work conflict $(r=0.267,0.200,0.200)$ based on time, pressure, and the three aspects of behavior $(P<$ $0.05)$. Depersonalization was positively correlated with work-family conflict pressure and behavior $(r=$ $0.328,0.325)$ and family-work conflict based on time, pressure and the three aspects of behavior $(r=$ $0.340,0.266,0.241 ; P<0.05)$. Personal achievement is negatively correlated with work-family conflict time and behavior $(r=-0.138,-0.100)$ and family-work conflict pressure and behavior $(r=-0.072,-0.078 ; P<0.05)$ (Table 3). 
Table 3

Correlation analysis between burnout and occupational stress and work-family-conflict $(n=1346)$

\begin{tabular}{|c|c|c|c|}
\hline Variable & Emotional exhaustion & Depersonalization & Personal accomplishment \\
\hline Work demands & $0.273^{\star *}$ & $0.162^{\star *}$ & $-0.128 * \star$ \\
\hline \multicolumn{4}{|l|}{ Work autonomy } \\
\hline Decision autonomy & $-0.104^{\star \star}$ & -0.017 & $-0.116 * *$ \\
\hline Working ability & $0.104^{\star *}$ & $-0.066^{\star}$ & $-0.166^{\star \star}$ \\
\hline \multicolumn{4}{|l|}{ Social support } \\
\hline Superior support & -0.049 & -0.036 & $-0.097 \star \star$ \\
\hline Colleague support & 0.027 & -0.051 & $-0.147 * \star$ \\
\hline Efforts & $0.284^{\star \star}$ & $0.179 * \star$ & $0.107^{\star * *}$ \\
\hline Reward & 0.036 & $-0.128 * \star$ & 0.018 \\
\hline Internal investment & $0.256 * \star$ & $0.138 * *$ & $0.083^{*}$ \\
\hline \multicolumn{4}{|l|}{ Work-family conflict } \\
\hline Time & $0.179 * \star$ & 0.010 & $-0.138 * \star$ \\
\hline Stress & $0.316 * \star$ & $0.328 * *$ & -0.050 \\
\hline Behavior & $0.308 * \star$ & $0.325^{\star \star}$ & $-0.100 * \star$ \\
\hline \multicolumn{4}{|l|}{ Family-work conflict } \\
\hline Time & $0.267 * \star$ & $0.340 * *$ & -0.052 \\
\hline Stress & $0.200 * \star$ & $0.266^{\star \star}$ & $-0.072^{\star \star}$ \\
\hline Behavior & $0.200 * \star$ & $0.241^{\star \star}$ & $-0.078 * \star$ \\
\hline
\end{tabular}

\section{Logistic regression analysis of factors affecting job burnout}

Single factor regression analysis was carried out using the degree of job burnout (No, Mild burnout $=0$, Moderate, Severe burnout $=1$ ) among the coal miners as a dependent variable and demographic variables (gender, ethnicity, age, length of service, education, type of work, shift system, marital status), various dimensions of occupational stress (work demands, decision autonomy, working ability, superior support, colleague support), efforts-reward dimensions (efforts, reward, internal investment), and the dimensions of work-family-conflict (work-family conflict, family-work conflict, and total score) as independent variables. The factors with $P<0.05$ in the univariate analysis were used as independent variables and incorporated into the Logistic regression equation for job burnout among the miners. The 
logistic regression independent variable assignment is shown in Table 4, and the logistic regression result is shown in Table 5.

Table 4

Individual factor assignment table

\begin{tabular}{|lll|}
\hline Predictor & Variable & Assignment \\
\hline X1 & Education & $\begin{array}{l}\text { Junior high school and below }=1 \text {, High school }=2 \text {, Bachelor and } \\
\text { college degree and above }=3\end{array}$ \\
\hline X2 & Type of work & $\begin{array}{l}\text { Anchor miners }=1, \text { Coal miners }=2 \text {, Excavate miners }=3, \\
\text { Moving frame miner }=4, \text { Other }=5\end{array}$ \\
\hline X3 & Marital status & Single $=1$, Married $=2$,Divorced $/$ Separated $=3$ \\
\hline X4 & Length of service & $1 \sim 10$ years $=1, \geq 11$ years $=2$ \\
\hline X5 & Age & $18 \sim 30$ years $=1,30 ~ 45$ years $=2,45 \sim 60$ years $=3$ \\
\hline X6 & Work demands & accurate value \\
\hline X7 & Work autonomy & accurate value \\
\hline X8 & Social support & accurate value \\
\hline X9 & Efforts & accurate value \\
\hline X10 & Reward & accurate value \\
\hline X11 & Internal investment & accurate value \\
\hline X12 & Work-family conflict & accurate value \\
\hline X13 & Family-work conflict & accurate value \\
\hline X14 & Total score for work- & accurate value \\
\hline
\end{tabular}


Table 5

The multivariate logistic regression analysis of the factors influencing job burnout in coal miners

\begin{tabular}{|lllllll|}
\hline Variable & Group & Beta & Wald & P & OR & 95\%Cl \\
\hline Length of service(years) & $18 \sim 30$ & & 22.807 & $<0.001$ & & \\
\cline { 2 - 7 } & $30 \sim 45$ & 0.717 & 22.686 & $<0.001$ & 2.048 & $1.525 \sim 2.750$ \\
\cline { 2 - 7 } & $45 \sim 60$ & 0.459 & 8.505 & 0.004 & 1.582 & $1.162 \sim 2.154$ \\
\hline Work demands & & 0.062 & 8.457 & 0.004 & 1.064 & $1.020 \sim 1.109$ \\
\hline Work autonomy & -0.074 & 33.823 & $<0.001$ & 0.929 & $0.906 \sim 0.952$ \\
\hline Efforts & 0.066 & 14.940 & $<0.001$ & 1.068 & $1.033 \sim 1.105$ \\
\hline Work-family conflict & & 0.025 & 5.324 & 0.021 & 1.025 & $1.004 \sim 1.047$ \\
\hline Total score for work-family-conflict & 0.073 & 45.760 & $<0.001$ & 1.076 & $1.054 \sim 1.099$ \\
\hline Constant & & -2.320 & 25.079 & $<0.001$ & 0.098 & \\
\hline
\end{tabular}

We used the Backward:wald method to introduce the above variables into the binary logistic regression equation. After 8 steps of regression, the Hosmer and Lemeshow test $\left(\chi^{2}=3.439, P=0.904\right.$; Nagelkerke $\left.R^{2}=0.170\right)$ indicated that the test model had a good fit. The risk factors for the variables that finally entered the equation are age $\left(\mathrm{OR}_{30 \sim 45}=2.048, \mathrm{OR}_{45 \sim 60}=1.582\right)$, work demands ( $\left.\mathrm{OR}=1.064\right)$, efforts ( $O R$ $=1.068)$, work-family conflict $(O R=1.025)$, and the total score for work-family-conflict $(O R=1.076)$, while the protective factor was work autonomy $(O R=0.929)$. This shows that advanced age, high work demands, more effort, and increased work-family-conflicts increases the risk of job burnout, while higher work autonomy is associated with low risk of job burnout.

\section{Discussion}

The results of this study showed that there was a difference in the total scores of job burnout among coal miners of different genders, ages, years of service, education, shift system, marital status, and types of work. Men's job burnout scores are higher than women's, 30-45 years old have the highest scores, and the group of 11 years old higher scores, higher education lower scores, shift miners scores are higher than day shifts, married/divorced scores are higher than single. Bhagavathula AS [24] conducted a survey of nursing center staff that also showed that men, single status and advanced age are risk factors associated with job burnout. The number of men working in coal mines is higher than that of women, and men tend to get heavier and more difficult workload than women. As a result, due to work-related factors, men tend to have more disregard for the work environment and the idea of easy escape from work than women [25]. Older workers with longer working years need to face family problems such as children's education, parental support, etc., and need to spend a certain amount of time and energy to balance family work problems. The double pressure of work and family makes older and longer-term employees 
prone to developing mental health diseases. On the other hand, young or newly hired employees are generally unmarried and childless, their parents are in good health, have fewer work tasks, and work pressure is lighter and easier to relieve [26]. Workers with lower academic qualifications in coal mining enterprises are generally engaged in positions with heavier manual labor, with poor work autonomy and high repetitiveness. On the other hand, those with higher academic qualifications are generally engaged in leadership or technical positions, and they can easily use the skills they have learned in their work, to get greater achievements and higher rewards. There is a difference in the burnout scores of workers who work in shifts compared to workers on regular day shifts. The risk of work stress for shift workers is higher than that of workers on regular working hours. The most direct damage to health due to working in shifts is sleep disturbance. Job burnout indirectly affects work performance [27]. People who work in shifts, especially those who work night shift, have poor sleep quality and health [28]. Long-term shift work is a high risk factor for metabolic syndrome, and is associated with increased incidence of cardiovascular disease and endocrine disease. People who work on night shifts cannot sleep normally at night like regular day shift workers. As a result of long-term shifts, the biological clocks of the workers tend to be disordered, since fatigue after work cannot be fully relieved and most of the working hours are more than 8 hours a day. Multiple factors such as long working hours and poor rest and relaxation can lead to high incidences of employee burnout [29]. The job burnout scores of unmarried coal miners is lower than that of married and divorced or widowed workers. The social roles of unmarried workers are relatively few. The married workers not only have responsibilities as employees, but they also have responsibilities towards their children, partners and parents. Consequently, these employees need to spend energy during working hours, as well as after working hours on family building, children's education or building relationships with their parents. As a result, reasonable release and relief from work-related stress and negative emotions cannot be obtained after work. Family pressure thus becomes a risk factor for burnout, with the risk being higher in older children [30, 31].

The results of the study show that the effort-reward imbalance is associated with the age, length of service, shift system and marital status of the miners. Workers with longer working years have been in the work position for a long time, and they are more familiar and serious about the work than those who have just started. In addition, in some tasks that new employees are unable to handle, older and long-term workers make more contributions. But there are some unavoidable objective problems, such as reaction ability, learning ability, adaptability, and physical health, compared with young workers who have just joined the job, there are still some differences between the leadership and the young workers. When new learning opportunities arise, young staff are generally the first to be considered. In addition, young staff with less seniority have more opportunities for promotion, which makes older workers with more experience feel like their efforts at work do not receive the attention and rewards they deserve, thus creating a greater psychological gap [32]. Since married workers are responsible for the economic wellbeing of their families, work income becomes very important for the working class, particularly for families with children. As a result, married workers invest more energy at work to complete their tasks smoothly to ensure the stability of income. The results of correlation analysis between job burnout and effort-reward imbalance show that emotional exhaustion is positively correlated with effort and internal 
investment. Depersonalization is positively associated with effort and internal investment, and negatively associated with return. Personal accomplishment is positively correlated with dedication and internal investment dimensions, indicating that the two are related. The effort-reward imbalance for dedication is closely associated with increased probability of high emotional exhaustion, depersonalization, and personal accomplishment [33, 17], is the most powerful predictor of emotional exhaustion and personal accomplishment [34]. A study by, Colindres $\mathrm{CV}$ found that after adjusting the income return of low-income workers, the imbalance of employee effort-reward was reduced, and the degree and incidence of job burnout were also alleviated [35].

The effort-reward imbalance is currently considered to be a major cause of work stress, with employees who have worked under effort-reward imbalance for a long time having obvious depression and burnout tendencies [36]. Most coal miners with low academic qualifications can only earn remuneration from manual labor, and it is easy to psychologically think that labor pay is not proportional to return. Maintaining a balance between workload and remuneration may enhance recovery during leisure time, improve sleep quality, and help reduce the rate of burnout [18].

There are differences in the occupational stress scores of coal miners of different genders, years of service, education, shift system and monthly income. Men are higher than women,the score of the group of $\geq 11$ years is higher than the group of $1-10$ years,the higher the education level and the higher the score,higher than shift work, miners with monthly income $>7000$ score the highest $(P<0.05)$. People with more working experience and high education tend to have higher incomes in coal mining enterprises, so they bear greater responsibilities and risks, and are more likely to have a sense of work tension. Day shift workers tend to have a larger workload than late shift workers. The results of job burnout and JCQ show that emotional exhaustion is positively associated with work demands and job skills, and negatively associated with decision-making autonomy, which are consistent with the results of a study by Persson $\mathrm{R}$ [37]. In addition, depersonalization is positively correlated with work demands and negatively correlated with job skills; personal accomplishment is negatively correlated with all dimensions of JCQ. The support obtained at work and the enjoyment of fair treatment have a protective effect on the occurrence of job burnout, while high work demands, low self-autonomy at work, large workload, low pay and job insecurity increase the risk of burnout [38]. Job burnout is closely associated with the working environment, the imbalance of payment and social support [39]. Increasing social support, increasing workers' sense of participation in work, setting a reasonable amount of work demands, and giving reasonable labor remuneration are conducive to reducing work-related negative emotions. It is easier for workers with type A personality to have negative emotions in unreasonable working environments. Negative work emotions aggravate job burnout and increase the risk of job burnout. A reasonable working system and working environment can enable people with perfect personalities to complete work tasks efficiently and with high quality, but unreasonable working environment, interpersonal relationships and work tasks make people with perfect personality traits suffer from a high degree of burnout [40].

Studies have shown that the model incorporating work-family-conflict comprehensively evaluates the health status of the occupational population [41]. The results of this study found that different shift 
systems and types of work influence the scores of work-family-conflict. Shift miners score higher than miners on day shifts; coal miners and excavate miners scores higher compared with other types of work $(P<0.05)$. This is probably because tunneling is the primary task of coal mining and its high work hazard coefficient lays the foundation for other work such as coal mining. As a tunneler, the work effort is also higher than other types of work, and the smooth completion of the work is of great significance to other coal operations. The correlation results show that the scores of other dimensions of conflicts, except for the time conflict between work and family, gradually increase as the degree of job burnout increases, indicating that the greater the work-family conflict, the greater the degree of job burnout [42, 43]. This is consistent with the findings of Shujuan Yang et al. that the work-family conflict among medical staff is positively correlated with job burnout [44], and demographic factors (sex,technical title,administrative duties) account for $5.4 \%$ of job burnout. The relationship between the scores of family-work conflict and emotional exhaustion and depersonalization are similar to those of other occupations, but it is opposite to the result of personal accomplishment [43];it may be that due to different occupations, doctors and other industries have higher family participation than coal miners after work. Therefore, family factors have a greater impact on the score of achievement reduction. Coal miners basically live in groups and go home less frequently. They basically participate in family activities and complete family role tasks through network communications. Emotional exhaustion and depersonalization reactions are higher when there is conflict between work and family, and the emotional performance is obvious. However, the completion of work tasks and obtaining a certain salary are the main ways for coal miners to perceive personal accomplishment. Therefore, the score of achievement reduction is somewhat different from the research results of other industries.

Regression analysis results show that age, work demands, effort, work-family conflict and total scores of work-family-conflict are risk factors for job burnout, and work autonomy is a protective factor. The results show that advanced age, higher work demands, more effort, and increased work-family-conflicts increase the risk of job burnout, while higher work autonomy is associated with low risk of job burnout. Smith TD and others [45] also found that the occurrence of job burnout is closely associated with gender, age, marital status, job content, family, coping style, social support, and other factors. Therefore, coal mining enterprises should pay attention to the mental health of the elderly and high paying workers. This is because the average age of the miners is high, and most of them are married with family responsibilities of supporting the elderly and raising young children. In addition, most of the miners are men, and the couples live apart for a long time, making them prone to emotional estrangement. It is difficult to obtain family support in time, making them prone to job burnout. Considering the influence family has on work, it is necessary to take care of family members, strengthen family bonds, and improve the happiness of the families of miners. At the same time, there is need to improve the autonomy of miners'work through health lectures and other forms, educate miners to use positive ways to relieve work pressure and prevent the occurrence of job burnout, conduct regular mental health evaluations of miners, guide miners with abnormal psychology, and promote corporate benefits.

\section{Conclusion}


Most Chinese coal miners have high job burnout. Age, work demands, effort and work-family-conflict are the risk factors for job burnout of coal miners while work autonomy is a protection factor. Our survey results emphasize that the management of coal mining enterprises must be aware of the risk factors of job burnout. The results show that advanced age, higher work demands, more effort, and increased workfamily-conflicts increase the risk of job burnout, while higher work autonomy reduces the risk of job burnout.

\section{Abbreviations}

JCQ:Job Content Questionnaire;ERI: Effort-Reward Inventory

\section{Declarations}

\section{Ethics approval and consent to participate}

The study was approved by the Ethical Committee of the First Affiliated Hospital of Xinjiang Medical University (No.20160218-109), and in accordance with the principles of the Declaration of Helsinki. All participants gave their written informed consent to take part in the present study.

\section{Consent for publication}

Not applicable.

\section{Availability of data and materials}

All data relevant to the study are included in the article or uploaded as online supplementary information. The datasets used and/or analysed during the current study are available from the corresponding author on reasonable request.

\section{Competing interests}

The authors declare that they have no conflicts of interest.

Funding this research was supported by the National Natural Science Foundation of China(grant No.81660533).

\section{Authors' contributions}

F.L.conceived and designed this study;H.D.and Xn.L.contributed equally to this work and are listed as the first coauthor;H.D.completed most of this paper in English; Xn.L.collected data and completed comments; Xn.L.,X.L.and F.L. provided many suggestions;X.A.,Z.M.,S.Z.and X.L.completed the input and analyzed the data.

\section{Acknowledgments}


The author would like to thank the personnel in charge of the coal mine who cooperated with us to obtain the information of the investigation and all coal workers who volunteered to participate in this investigation.

\section{References}

1. Freudenberger HJ.Staff burnout.Journal of Social Issues.1974;30:159-165. (https://dx.doi.org/10.1111/ j.1540-4560.1974.tb00706.x.)

2. Yoo Jung Kim, Erik Faber.What medicine can teach academia about preventing burnout.Nature.2019. (https://dx.doi.org/10.1038/d41586-019-01451-9.》

3. Maslach C,Jackson S E,Leiter M P.Maslach Burnout Inventory Manual.Consulting Psychologists Pr.1996.

4. Adriaenssens J,De Gucht,Véronique,et al.Determinants and prevalence of burnout in emergency nurses: A systematic review of 25 years of research. International Journal of Nursing Studies.2015;52:649-661. (https://dx.doi.org/10.1016/j.jijnurstu.2014.11.004.)

5. De Maeyer C, Schoenmakers B.Exploring intergenerational differences in burnout and how they relate to work engagement, norms, and values:a mixed-methods study. BJGP Open.2019;3,2. (https://dx.doi.org/ 10.3399/ bjgpopen18X101637.)

6. Chemali Z,Ezzeddine F L,Gelaye B,et al.Burnout among healthcare providers in the complex environment of the Middle East:a systematic review. BMC Public Health.2019;19:1337. (https://dx.doi.org/10.1186/ s12889-019-7713-1.)

7. Dubale BW,Friedman LE,Chemali Z,et al.Systematic review of burnout among healthcare providers in sub-Saharan Africa. BMC Public Health.2019;19,1247.(https://dx.doi.org/10.1186/s12889-019-75667.)

8. Yilmaz A.Burnout,job satisfaction, and anxiety-depression among family physicians: Acrosssectionalstudy. J Family Med Prim Care.2018;7,952-956. (https://dx.doi.org/10.4103/jfmpc.jfmpc_59_18.)

9. Yong $X, G a o X, Z$ hang Z,et al.Associations of occupational stress with job burn-out, depression and hypertension in coal miners of Xinjiang,China: a cross-sectional study.BMJ Open.2020; 10,e036087. (https://dx.doi.org/10.1136/bmjopen-2019-036087.)

10. Fu-Dong Liu,Zi-Qiang Pan,Sen-Lin Liu,et al.The Estimation of the Number of Underground Coal Miners and Normalization Collective Dose at Present in China.Radiat Prot Dosimetry.2017;174,302307. (https:// dx.doi.org/10.1093/rpd/ncw146.)

11. Li Liu,Lie Wang,Jie Chen.Prevalence and Associated Factors of Depressive Symptoms among Chinese Underground Coal Miners.BioMed Research International Volume.2014;2014:987305. (https://dx.doi.org/ 10.1155/ 2014/987305.)

12. LuY,Zhang Z,Gao S,et al.The Status of Occupational Burnout and Its Influence on the Psychological Health of Factory Workers and Miners in Wulumuqi,China. BioMed Research 
International.2020;2020:6890186. (https://dx.doi.org/10.1155/2020/6890186. )

13. Mingming Deng,Feng Wu,Jun Wang,et al.Musculoskeletal disorders, personality traits, psychological distress, and accident proneness of Chinese coal miners.Work.2017;57,441-449. (https://dx.doi.org/ 10.3233/WOR-172569.)

14. Min Yu, Jizu Li.Psychosocial safety climate and unsafe behavior among miners in China: the mediating role of work stress and job burnout.Psychol Health Med.2020;25:793-801. (https://dx.doi.org/10.1080/ 13548506. 2019.1662068.)

15. Shujuan Yang,Danping Liu,Hongbo Liu,et al.Relationship of work-family conflict, self-reported social support and job satisfaction to burnout syndrome among medical workers in southwest China: A cross-sectional study.PLoS One.2017;12:e0171679.(https://dx.doi.org/10.1371/journal.pone. 0171679. )

16. Martini M, Converso D. Burnout study in the healthcare: relationship with the patients and workfamily relationship as demands and resources.G Ital Med Lav Ergon.2012;34,A41-50.

17. Tang $L$, Pang $Y, H e Y$, et al.Burnout among early-career oncology professionals and the risk factors. Psychooncology.2018;27,2436-2441.(https://dx.doi.org/10.1002/pon.4847.)

18. Gluschkoff K, Elovainio M, Kinnunen U,et al.Work stress, poor recovery and burnout in teachers.Occup Med (Lond). 2016;66,564-70. (https://dx.doi.org/ 10.1093/ occmed/kqw086. )

19. Fuye Li, Jiwen Liu,Yulong Lian,et al.Reliability and validity of job burnout measurement tool in knowledge worker.Chinese Journal of Occupational Diseases of Labour hygiene.2009;27,156-159. (In Chinese).

20. Fuye Li.A study on the relationship between occupational stress and job burnout and its influencing factors in knowledge worker. Xinjiang Medical University. 2008;25-29.(In Chinese).

21. Wang Li,Li Hua,Gao Hongping, et al. A Reliability and Validity Analysis of Chinese Version of Job Content Questionnaire for Health Professionals. Journal of Baotou Medical College. 2010;26,20-22. (In Chinese).

22. Liu Baoying,Su Liqun,Yang Hua,et al. Reliability and validity verification of the job content questionnaire (JCQ22)The second time National Conference on Occupational Psychology and Tension.2007.(In Chinese).

23. Carlson D S,Kacmar K M,Wayne $\mathrm{J} \mathrm{H}$,et al. Measuring the positive side of the work-family interface: Development and validation of a work-family enrichment scale.Journal of Vocational Behavior.2006; 68,131-164.

24. Bhagavathula AS, Abegaz TM, Belachew SA,et al.Prevalence of burnout syndrome among healthcare professionals working at Gondar University Hospital, Ethiopia.J Educ Health Promot.2018;7:145. (https:// dx.doi.org/10.4103/jehp.jehp_196_18.)

25. Banerjee S, Califano R, Corral J, et al. Professional Burnout in European Young Oncologists: Results of The European Society For Medical Oncology (ESMO)Young Oncologists Committee Burnout Survey. Annals of Oncology Official Journal of the European Society for Medical Oncology. 2017;28:1590-1596.(https:// dx.doi.org/10.1093/annonc/mdx196.) 
26. Shanafelt T D, Hasan O, Dyrbye L N, et al. Changes in Burnout and Satisfaction With Work-Life Balance in Physicians and the General US Working Population Between 2011 and 2014. Mayo Clinic Proceedings.2015; 90,1600-1613.(https://dx.doi.org/10.1016/j.mayocp.2015.08.023.)

27. Giorgi F, Mattei A, Notarnicola I, et al. Can sleep quality and burnout affect the job performance of shift work nurses? A hospital cross:ectional study. Journal of Advanced Nursing.2018;74,698-708. (https://dx.doi.org/ 10.1111/jan.13484.)

28. Leser C, Tisch A, Tophoven S.Shift Work among Men and Women on the Threshold to Higher Working Age-Working Conditions and Health Status.Gesundheitswesen.2016;78,765-771. (https://dx.doi.org/10.1055/ s-0034-1396850. )

29. Dall'Ora,Chiara,Ball J, et al. Characteristics of shift work and their impact on employee performance and wellbeing: A literature review. International Journal of Nursing Studies.2016;57,12-27. (https://dx.doi.org/ 10.1016/j.jijurstu.2016.01.007.)

30. Perkins J M, Lee H Y, James K S, et al. Marital status, widowhood duration, gender and health outcomes: a cross-sectional study among older adults in India. BMC Public Health.2016;16:1032. (https://dx.doi.org/ 10.1186/s12889-016-3682-9.)

31. Carlson D L.Deviations From Desired Age at Marriage: Mental Health Differences Across Marital Status. Journal of Marriage and Family.2012;74,743-758.(https://dx.doi.org/10.1111/j.17413737.2012.00995.x)

32. Huang Li. Association study of job stress,burnout and presenteeism in health care workers.Fudan University. 2013. (In Chinese)

33. Liza J, Jonathan H, Roslyn T. Effort-reward imbalance and burnout among humanitarian aid workers. Disasters.2018;43:67-87.(https://dx.doi.org/10.1111/disa.12288.)

34. Guo HQ, Guo HF, Yang Y,et al.Internal and External Factors Related to Burnout among Iron and Steel Workers: A Cross-Sectional Study in Anshan, China.PLoS One.2015;10:e0143159.(https://dx.doi.org/ 10.1371/journal.pone.0143159.)

35. Colindres C V, Bryce E, Coral-Rosero P, et al. Effect of effort-reward imbalance and burnout on infection control among Ecuadorian nurses. International Nursing Review.2017;65:190-199. (https://dx.doi.org/ 10.1111/inr.12409. )

36. JiY Q, Li S, Wang J, et al. Association of occupational stress with job burnout and depression tendency in workers in Internet companies. Chinese journal of industrial hygiene and occupational diseases.2018; 36,241-246.(https://dx.doi.org/10.3760/cma.j.issn.1001-9391.2018.04.001.)

37. Persson R,sterberg, Kai, Viborg N, et al. Two Swedish screening instruments for exhaustion disorder: cross-sectional associations with burnout, work stress, private life stress, and personality traits.Scandinavian Journal of Public Health.2017;45:381-388. (https://dx.doi.org/10.1177/1403494817696182.)

38. Aronsson G, Theorell T, Grape T, et al. A systematic review including meta-analysis of work environment and burnout symptoms. BMC Public Health.2017;17:264. (https://dx.doi.org/10.1186/s12889-017-4153-7.) 
39. Renzo Bianchi.Burnout is more strongly linked to neuroticism than to work-contextualized factors. Psychiatry Res.2018;270,901-905.(https://dx.doi.org/10.1016/j.psychres.2018.11.015.)

40. Robakowska M, Tyrańska-Fobke A, Walkiewicz M, et al. Adaptive and maladaptive perfectionism, and professional burnout among medical laboratory scientists. Medycyna Pracy. 2018;69:253-260. (https:// dx.doi.org/10.13075/mp.5893.00644.)

41. Chen Yidi, Gan Yiqun. Harmonious personality, social support, self-control, and work-family conflict: Comparison of three models based on occupational groups.Journal of Peking University. (Natural Science Edition), 2018;54:1123-1132. (In Chinese)

42. Wu G, Wu Y, Li H, et al.Job Burnout, Work-Family Conflict and Project Performance for Construction Professionals: The Moderating Role of Organizational Support.Int J Environ Res Public Health.2018; 15:2869.(https://dx.doi.org/10.3390/ijerph15122869.)

43. Chen L, Liu J, Yang H, Ma H, et al.Work-family conflict and job burn-out among Chinese doctors: the mediating role of coping styles.Gen Psychiatr.2018;31:e000004.(https://dx.doi.org/10.1136/gpsych2018-000004.)

44. Shujuan Yang, Danping Liu, Hongbo Liu,et al.Relationship of work-family conflict, selfreported social support and job satisfaction to burnout syndrome among medical workers in southwest China: A cross-sectional study.PLoS One.2017;12:e0171679.(https://dx.doi.org/10.1371/journal.pone. 0171679.)

45. Smith TD,Dejoy D M,Dyal M A,et al.Impact of work pressure,work stress and work-family conflict on fire fighter burnout. Arch Environ Occup Health.2019;74(4):215-222. (https://dx.doi.org/10.1080/19338244. 2017.1395789.) 